\title{
A STOCHASTIC MODEL OF MIGRANT RESCUE IN THE MEDITERRANEAN SEA ${ }^{1}$
}

\author{
Amitrajeet A. BATABYAL \\ Department of Economics, Rochester Institute of Technology, 92 Lomb Memorial Drive, Rochester, \\ NY 14623-5604, USA. \\ aabgsh@rit.edu \\ Hamid BELADI \\ Department of Economics, University of Texas at San Antonio, One UTSA Circle, San Antonio, TX \\ 78249-0631, USA. \\ Hamid.Beladi@utsa.edu
}

\begin{abstract}
We theoretically study the rescue of destitute migrants seeking to reach Europe on boats across the Mediterranean Sea. To this end, we first construct a discrete-time Markov chain (DTMC) model of a stylized rescue process. Second, we specify the one-step transition probabilities of the DTMC. Third, we delineate a recursive algorithm that can be used to compute the limiting or steady-state probabilities of our DTMC model. Finally, we use these limiting probabilities to compute the average throughput. This metric is an efficiency measure and it tells us the long run expected number of migrants that are rescued by the stylized rescue process under study.
\end{abstract}

Keywords: Markov Chain, Mediterranean Sea, Migrant, Rescue, Uncertainty

JEL classification: J61, D81

\section{Introduction}

Grinding poverty and economic mismanagement in many parts of sub-Saharan Africa combined with political turmoil in nations such as Libya have led many Africans to seek a better life in Europe. In addition, the ongoing war in Syria, political and sectarian tensions across the Middle East, and the rise of the so called Islamic State have similarly left many people desirous of a better life in Europe and in other parts of the rich world.

As noted by The Economist (Anonymous $(2014,2015)$ ), the above two broad forces have now resulted in large numbers of migrants making the perilous journey to southern European nations across the Mediterranean Sea in flimsy boats from points in North Africa such as Libya and Turkey. As noted by D'Emilio and Corder (2015) and by Phillip (2015), this journey across the Mediterranean Sea on unstable vessels can be very dangerous. Therefore, it is not surprising that nearly 2,000 migrants have perished attempting to cross the Mediterranean Sea from Africa in a desperate bid for asylum or work in Europe. ${ }^{2}$

The European Union (EU) has been slow to respond to the migration crisis that has led to thousands of deaths at sea. However, as pointed out by Kanter (2015), there is now agreement among EU leaders that there needs to be a significantly more comprehensive response to deal not only with the migrant smugglers but also with the migrants themselves. A key part of this comprehensive response seeks to stop smugglers with human cargo before they leave the shores of North African nations such as Libya. This notwithstanding, authorities in front-line nations such as Italy have increasingly begun to rescue the hapless migrants, following which, they are brought to appropriate processing points on Italian islands such as Lampedusa and Sicily.

This migrant crisis has become a major public policy problem not only in the front-line nations of Europe such as Greece and Italy but also in more northern nations such as Germany and Great Britain. Despite the salience and the contemporary relevance of this migrant crisis,

\footnotetext{
${ }^{1}$ Batabyal acknowledges financial support from the Gosnell endowment at RIT. The usual absolution applies.

${ }^{2}$ See Pianigiani (2015) and Yardley and Povoledo (2015) for additional details on these points.
} 
to the best of our knowledge, there are no theoretical analyses of any aspect of this problem in the extant literature. As best as we can tell, the only paper that has addressed the related problem of external border enforcement is by Haake et al. (2013). These researchers use a numerical example to show that when the EU Commission takes an active role, under certain circumstances, it can put in place the optimal level of border control by member states.

Given the absence of theoretical research on the above described migrant problem, our objective in this note is to commence the theoretical study of the migrant problem by concentrating on a particular aspect of this problem. Specifically, we focus on the rescue of impoverished migrants - attempting to cross the Mediterranean Sea from a point in North Africa or Turkey - by the naval authorities of a front-line nation such as Italy. The rest of this note is organized as follows. Section 2 constructs a discrete-time Markov chain (DTMC) model of a stylized rescue process. Section 3 specifies the one-step transition probabilities of the DTMC. Section 4 delineates a recursive algorithm that can be used to compute the limiting or the steady-state probabilities of our model. Section 5 uses these limiting probabilities to compute the average throughput. This last metric is an efficiency measure and it tells us the long run expected number of migrants that are rescued by the stylized rescue process we analyze. Section 6 concludes and then discusses two ways in which the research in this note might be extended.

\section{The Theoretical Framework}

Consider a flimsy vessel such as a rubber dinghy in the Mediterranean Sea that is loaded with a large number of migrants who are seeking to reach the shores of a southern European nation such as Italy. In the course of its journey across the Mediterranean Sea, this dinghy is first spotted and then approached by a navy ship from a front-line nation such as Italy. The goal of this navy ship is to rescue the migrants from their presently precarious rubber dinghy. To this end, the ship lays a bridge to the vessel loaded with the migrants.

Migrants are rescued one at a time and we suppose that the transfer of a migrant from the rubber dinghy to the navy ship occurs at the beginning of a time slot. These time slots are of unit length and the time it takes to transfer a migrant from the rubber dinghy to the navy ship is one time slot. What adds uncertainty to the rescue process is that the systematic transfer of migrants from the rubber dinghy to the navy ship may fail because of a variety of reasons. For instance, a migrant being transferred may be too weak to travel over the bridge and hence may fall into the sea. Alternately, instead of moving to the navy ship in an orderly manner (one migrant per time slot), migrants, fearful of the rubber dinghy sinking, may rush the bridge and attempt to board the rescuing navy ship all at once. Finally, some migrantspotentially children - may not understand that they are to move to the navy ship one at a time and therefore they may attempt to board the navy ship along with their parents and/or relatives.

We model the above aspects of the rescue process by supposing that each migrant transfer from the rubber dinghy to the navy ship may fail with some probability $\in>0$.A failed migrant transfer is attempted again at the beginning of the next time slot. The numbers of migrants arriving on the navy ship during the various time slots are independent random variables with a common discrete distribution function given by $\left\{b_{j}, j=0,1,2, \ldots\right\}$. Newly arriving migrants are all housed in the rescuing navy ship. To keep the subsequent mathematical analysis tractable, we suppose that the mean arrival rate of the migrants is smaller than the mean number of attempts needed to transfer a migrant successfully from the rubber dinghy to the navy ship.

Mathematically, this means that the inequality $\sum_{\forall j} j b_{j}<1 / \in$ holds.

Let $Z_{n}$ denote the number of migrants in the rubber dinghy/navy ship system at the beginning of the $n t h$ time slot. Then, it follows that the stochastic process $\left\{Z_{n}\right\}$ is a discrete- 
time Markov chain with state space given by the set $I=\{0,1,2, \ldots\} .^{3}$ With this theoretical framework in place, our next task is to specify the one-step transition probabilities of the DTMC model that we have just described.

\section{One-Step Transition Probabilities}

It helps to combine the one-step transition probabilities of our DTMC model into two groups. For the first group, we focus on the states $j=0,1,2, \ldots$ For the second group, we concentrate on the states for which $i \geq 1$ and $j=i-1, i, i+1, \ldots$ Putting these two groups of states together, the relevant one-step transition probabilities are

$$
p_{0 j}=b_{j}, \text { for } j=0,1,2, \ldots
$$

and

$$
p_{i j}=\in b_{j-i}+(1-\in) b_{j-i+1}, \text { for } i \geq 1, j=i-1, i, i+1, \ldots,
$$

with the understanding that $b_{-1}=0$. Having specified the one-step transition probabilities, we now proceed to describe a recursive algorithm that can be used to compute the limiting or steady-state probabilities of our model.

\section{A Recursive Algorithm}

The captain of our navy ship or, more generally, an appropriate EU asylum and/or immigration authority may wish to compute the limiting probabilities for the DTMC migrant rescue model that we are studying. To see how this computation exercise might actually be undertaken, let us first use definition 3.3.2 in Tijms (2003, p. 98) and theorem 4.1 in Ross (2014, p. 206) to point out that for our DTMC model $\left\{Z_{n}\right\}$ with state space $I=\{0,1,2, \ldots\}$, the probability distribution $\left\{\pi_{j}, j \in I\right\}$ is the limiting or steady-state distribution if

$$
\pi_{j}=\sum_{k \in I} \pi_{k} p_{k j}, j \in I \text {. }
$$

Now, note that for the DTMC model under study here, the one-step transition probabilities $p_{i j}=0$ as long as $j \leq i-2$. In addition, for any state $i \neq 0$, the mean number of transitions per unit time out of the set $B=\{i, i+1, i+2, \ldots\}$ ought to equal the mean number of transitions per unit time into the same set $B$. Putting the above two pieces of information together, we obtain an equation that provides us with the recursive algorithm that we seek. Specifically, we get

$$
(1-\in) b_{0} \pi_{i}=\sum_{k=0}^{k=i-1} \pi_{k}\left[\in \sum_{l=i-k}^{l=\infty} b_{l}+(1-\in) \sum_{l=i-k+1}^{l=\infty} b_{l}\right], \text { for } i=1,2,3, \ldots
$$

We now proceed to our final task in this note. This involves using the limiting probabilities that we have just described to calculate the average throughput. Note that we are interested in this metric because it is an efficiency measure and it gives us a closed-form expression for the long run expected number of migrants that are rescued by the stylized rescue process under study.

\section{The Average Throughput}

There are two ways to calculate the average throughput associated with the migrant rescue process that we are studying. First, by direct computation, the average throughput is $\sum_{j=1}^{\infty} \pi_{j} \times(1-\in)$. This expression can be further simplified and this simplification gives us

\footnotetext{
${ }^{3}$ See standard textbooks such as Tijms (2003, pp. 83-84) or Ross (2014, pp. 183-184) for a more detailed corroboration of this claim
} 


$$
\text { Average Throughput }=\sum_{j=1}^{j=\infty} \pi_{j} \times(1-\epsilon)=\left(1-\pi_{0}\right)(1-\epsilon)
$$

Second, the average throughput can also be calculated by recognizing that this metric is, in fact, equal to the mean input into our DTMC model of migrant rescue ${ }^{4}$. Now, if we denote the mean input by $\delta$ then it follows that $\delta=\sum_{\forall k} k b_{k}$. Therefore, we have $\left(1-\pi_{0}\right)=\delta /(1-\in)$ or, rearranging, the average throughput is, once again, equal to $\left(1-\pi_{0}\right)(1-\epsilon)$.

Inspecting the right-hand-side (RHS) of equation (5), we see that the long run expected number of migrants that are rescued by the stylized rescue process under study (the average throughput) is given by the product of two terms. The first term $\left(1-\pi_{0}\right)$ can be interpreted as the long run fraction of migrants who are waiting to be rescued by the navy ship. The second term $(1-\in)$ is the probability that an individual migrant is transferred from the rubber dinghy to the navy ship without incident. Since the average throughput is a measure of the efficiency of our migrant rescue process, equation (5) tells us that this efficiency can be increased by making $\pi_{0}$ and/or $\in$ small. From a practical perspective, the limiting probability $\pi_{0}$ is unlikely to be controllable by, say, the captain of the navy ship. However, the failure probability $\in$ certainly ought to be a control variable for the navy ship captain. Therefore, the smaller is $\in$, the greater is the efficiency of our migrant rescue process. In particular, in the limit as $\in \rightarrow 0$, the efficiency of the migrant rescue process is as high as it can possibly be.

\section{Conclusions}

In this note, we theoretically studied the rescue of destitute migrants seeking to reach Europe on boats across the Mediterranean Sea. To this end, we first constructed a discretetime Markov chain (DTMC) model of a stylized rescue process. Second, we specified the one-step transition probabilities of the DTMC. Third, we delineated a recursive algorithm that could be used to compute the limiting or steady-state probabilities of our model. Finally, we used these limiting probabilities to compute the average throughput which is an efficiency measure. This metric gave us the long run expected number of migrants that were rescued by the stylized rescue process under study.

The analysis in this note can be extended in a number of different directions. In what follows, we suggest two possible extensions. First, it would be useful to model an alternate scenario in which an access control rule is used to manage the migrants. Specifically, there would now exist an inland "gate" and this gate would be closed to newly arriving migrants when the number of migrants awaiting processing has reached some threshold. Once the number of migrants to be processed declines to some level below the threshold, this gate would be opened again. Second, it would also be instructive to analyze a setting in which with some positive probability, a migrant is denied asylum or a safe haven in an EU nation because (s)he is deemed to be, for instance, a security risk. Studies that analyze these aspects of the underlying problem will provide additional insights into the nexuses between alternate ways of apprehending and processing migrants and the working of migrant rescue schemes.

\section{References}

Anonymous. 2014. A surge from the sea: Illegal migration is causing strains across the continent, The Economist, August 16.

Anonymous. 2015. Europe's boat people: For those in peril, The Economist, April 25.

D'Emilio, F., and Corder, M. 2015. Italy: At least 40 migrants dead at sea, 320 others rescued, Washington Post, August 15.

\footnotetext{
${ }^{4}$ This statement is related to a well known result in queuing theory known as Little's formula. For textbook discussions of this formula, see Tijms (2003, pp. 50-53) or Ross (2014, pp. 482-483).
} 
Haake, C.J., Krieger, T., and Minter, S. 2013. On the institutional design of burden sharing when financing external border enforcement in the EU, International Economics and Economic Policy, 10, 583-612.

Kanter, J. 2015. E.U. agrees to naval intervention on migrant smugglers, New York Times, May 18.

Phillip, A. 2015. Watch the 'heart-stopping' moment rescuers pull drowning migrants out of the Mediterranean Sea, Washington Post, August 6.

Pianigiani, G. 2015. Hundreds of migrants rescued after boat capsizes off Libya, New York Times, August 5.

Ross, S.M. 2014. Introduction to Probability Models, 11th edition. Academic Press, Amsterdam, The Netherlands.

Tijms, H.C. 2003. A First Course in Stochastic Models. Wiley, Chichester, UK.

Yardley, J., and Povoledo, E. 2015. Mediterranean shipwreck kills 40 before a rescue, New York Times, May 5. 